\title{
Practice of Contact Lens During COVID-19
}

\author{
Janak Poudel ${ }^{1 *}$ and Premnath Krishnasamy ${ }^{2}$ \\ ${ }^{1}$ B .Optom, Vittala International Institute of Ophthalmology, India \\ ${ }^{2}$ M. Optom, Assistant Professor, Vittala International Institute of Ophthalmology, \\ India \\ *Corresponding Author: Janak Poudel, B .Optom, Vittala International Institute of \\ Ophthalmology, India.
}

Received: November 18, 2020

Published: November 30, 2020

(C) All rights are reserved by Janak Poudel

and Premnath Krishnasamy.

\section{Abstract}

A novel corona virus ( $\mathrm{CoV}$ ), the severe Acute Respiratory syndrome coronavirus-2 (SARS-CoV-2), results in the coronavirus disorder 2019 (COVID-19). The SARS-CoV-2 has been detected in the tears and conjunctival secretions in COVID-19 patients with conjunctivitis. Till date there is no proof that contact lens wearers are more likely to contract COVID-19 than spectacle wearers. The virus is known to be transferred through hand contact, and thus could be transferred to contact lenses throughout their application and removal. Five essential areas of action applicable in CL exercise to reduce the transmission of COVID-19 are: Patient management, personal protective equipment, disinfection of CL equipment and CL trail set, hand hygiene, CL practitioner and staff monitoring. Although the pandemic is increasing day by day, as optometrists should provide the care as well as services to the needy people following the recommended guidelines.

Keywords: COVID-19; Corona Viruses; Contact Lens (CL); Optometrist; Hand Hygiene; Disinfection; Respiratory; Practitioner

\section{Introduction}

A novel corona virus ( $\mathrm{CoV}$ ), the severe Acute Respiratory syndrome coronavirus-2 (SARS-CoV-2), results in the coronavirus disorder 2019 (COVID-19) [1]. Coronaviruses were named according to their structure under microscope. The virus includes a center of genetic fabric surrounded through an envelope with protein spikes. This gives it the appearance of a crown. Corona means "crown" in Latin [3].

Coronaviruses can cause respiratiory illnesses or gastrointestinal illnesses. Respiratory illnesses can vary from a common cold to a more extreme illnesses e.g. Middle East Respiratory Syndrome (MERS -CoV), Severe Acute Respiratory syndrome (SARS-CoV). According to the United States Center for Disease Control and Prevention and the European Centre for Disease Prevention and Control, virus transmission is occuring via human-to-human [1] The proof from different coronaviruses and respiration illnesses suggests that the disorder may also unfold via massive respiratiory droplets and direct or indirect touch with inflamed secretions. The droplets can make contact with the nose, mouth, eyes, or upper respiration tract of every other individual via 3 essential critical routes. The first is an airborne transmission in case of close contact among human beings (approximately 6 feet). The second is an instantaneous touch transmission as when two people shake their hands and the contaminated hand then touches a risk area on the second person. The third source is an indirect contact transmission where an infected individual touches an object that is then touched by the second one individual. Also it is known that its incubation duration is between 2 to 14 days [1-4,12-15].

Additionally, a problem for ophthalmic practitioners is that SARS-CoV-2 has been detected in the tears and conjunctival secretions in COVID-19 patients with conjunctivitis [4,5,14]. Till date there is no proof that contact lens wearers are more likely to contract COVID-19 than spectacle wearers. The virus is known to be transferred through hand contact, and thus could be transferred to contact lenses throughout their application and removal [4,17-19]. In one report, positive tear and conjunctival secretions occurred in 
a single affected person who developed conjunctivitis from a cohort study of 30 sufferers with a novel coronavirus [8]. In another report, 64 samples of the tear film from 17 sufferers with COVID -19 confirmed no proof of SARS-CoV-2 through viral culture or reverse transcriptase polymerase chain reaction (RT-PCR) $[4,11]$. Further the frequency of conjunctivitis in patients with COVID-19 stated thus far is less, even though it has been recommended that CoVs is also transmitted through aerosol touch with the conjunctiva in sufferers with active disease [4].

\section{Areas of action}

Looking at available proof in research papers, from the British Contact Lens Association and US Center for Disease Control and Prevention and the European Centre for Disease Prevention and Control, there are 5 essential areas of action applicable in CL practice to reduce the transmission of COVID-19, they are: Patient management, personal protective equipment, disinfection of CL equipment and CL trail set, hand hygiene, CL practitioner and staff monitoring [19,21].

\section{Patient management}

Firstly, in terms of patient management, patient and CL practitioner contacts should be less. The practitioner should work to manage cases within an optometric framework, which includes telephone contact with patients reporting contact lens problems and/ or a video consultation using a mobile phone app to enable rapid triage and management as well as to explore the possibility of rescheduling non-urgent appointments. In the case of appointments, it is important to note down some history regarding the patient travelling history within 14 days, patient with upper respiratory tract infection, and patient with conjunctivitis. If patients are deemed to have increased risk factors then it is more important to postpone the CL appointments for at least 14 days, which is the incubation period of COVID-19 [16,19,21,25]. The practitioner can decide to instruct the patient to stop CL in case they are using them.

\section{Personal protective equipment}

Personal protective equipment together with goggles or face shield, water-proof gloves and N95 masks should be used to lessen the chance of contamination through both airborne and direct transmission. As well as for the protection measures we can use shields on slit lamp [12-16].

\section{Disinfection}

The disinfection of CL equipment and CL trial set is an important area in CL practice to reduce the transmission of COVID-19. A thorough cleaning of devices used in CL practice together with trail frame, trail lenses, head and chin rest of slit lamp, keratometer can be carried out correctly with disinfectants together with sodium hypochlorite, 70\% iso-propyl alcohol. This has to be carried out as soon as the person has left the room. In case of specialty CL fitting such as RGP in keratoconus, OrthoK, and scleral, where a trail set of CLs are used, it becomes very important to ensure that CL are disinfected well. All CL accessories (suction holder, plunger, etc.) need to be cleaned with soap and water and replaced periodically and if possible, their use may be avoided [25].

\section{Hand hygiene}

Hands are a common vector for the transmission of respiratory infection, so hand sanitization must be performed through hand washing, before and after contact with any patient as well as before performing the test. Hands can be sanitized through the cleaning hands with an alcohol based hand rub or with cleaning soap and water or both. It is important that during patient examination, CL practitioner must avoid touching their own face, nose, mouth and eyes. Patient should also be informed about hand hygiene as well as cleaning of contact lens before using and not using disposable CLs longer than they are intended [4,13,15-22].

\section{The CDC and WHO advice all of us to}

- Wash their fingers frequently with cleaning soap and water for as a minimum 20 seconds.

- If cleaning soap and water are not readily available, they should use hand sanitizer that contains at least $60 \%$ alcohol. They should follow the WHO hand wash guideline

- They ought to keep away from touching their eyes, nose, and mouth with unwashed fingers [19,22].

\section{Cl practitioner and personnel monitoring}

Travelling history among staff should be noted down as well as CL practitioners and staff with potential condition of flu-like symptoms should not be attending work [19].

\section{How to deal with follow up cases}

Although the pandemic is increasing day by day, we as optometrists should provide the care as well as services to the needy people. Those patients who want to be updated about their contact lens care and maintenance steps, a soft copy of the instructions can be forwarded to these patients. Follow up examinations should be done only if they are an absolute must. Tele consulting can be attempted and the patients may be taught to take the picture of the eye with lens on by using educational videos [23-25]. 
Disinfection of Multi- person use touch lens

Gas permeable

- $\quad$ Step 1: Place 3\% hydrogen peroxide with GP lens in a nonneutralizing case.

- $\quad$ Step 2: Disinfect lens for 3+ hours.

- $\quad$ Step 3: Rinse GP lens with Multipurpose Solution (MPS). Pat dry, store dry.

Hybrid and soft

- $\quad$ Step 1: Place 3\% hydrogen peroxide with soft or hybrid lens in non-neutralizing case for 3 +hours.

- $\quad$ Step 2: Transfer soft or hybrid lens to a neutralizing case. Fill with fresh $3 \%$ hydrogen peroxide. Add neutralizing disc or tablet as recommended by manufacturer.

- $\quad$ Step 3: Neutralize lens for 6+ hours, or as directed by manufacturer [20].

\section{Conclusion}

In conclusion, so far no proof indicates that contact lens wearers who are asymptomatic should stop contact lens wear due to the risk of developing COVID-19, that wearing prescription spectacles affords safety from contracting SARS-CoV-2, or that any form of contact lens material is much more likely to increase or lessen the danger of COVID-19 infection. Practitioners should focus on being complaint about the recommended protocols for CL lens fitting and case especially during the peak of the pandemic, to avoid being the cause of transmission. Patients should be reminded of the need to dispose daily disposable lenses upon removal, the need for suitable disinfection with reusable lenses, together with using a rub and rinse step in such lenses where indicated, and suitable case cleansing and replacement. Finally, if the patient has an episode of cold or flu like symptoms, they need to discontinue wearing lenses and resume only $24 \mathrm{~h}$ after the symptoms resolve. The pair in use should be discarded safely as medical waste. As of now daily disposable lenses do not offer any additional safety from COVID 19, but can be worn for obvious advantages of disposability.

\section{Bibliography}

1. Coronavirus Disease (COVID-19).

2. G Miller. Researchers are tracking another pandemic, too of coronavirus misinformation (2020).

3. Y Chen., et al. "Structure analysis of the receptor binding of 2019-nCoV". Biochemical and Biophysical Research Communications 525.1 (2020): 135-140.
4. L Jones., et al. "The COVID-19 pandemic: Important considerations for contact lens practitioners". Contact Lens and Anterior Eye 43.3 (2020): 196-203.

5. Y Peng and YH Zhou. "Is novel coronavirus disease (COVID-19) transmitted through conjunctiva?" Journal of Medical Virology (2020).

6. L Seah and R Agarwal. "Can the Coronavirus Disease 2019 (COVID-19) Affect the Eyes? A review of coronaviruses and Ocular Implications in Humans and Animals". Ocular Immunology and Inflammation (2020): 1-5.

7. AA Axari and NP Barney. "Conjuctivitis: a systemic review of diagnosis and treatment". JAMA 310.16 (2013): 1721-1729.

8. J Xia., et al. "Evaluation of coronavirus in tears and conjunctival secretions of patients with SARS-CoV-2 infection". Journal of Medical Virology 92.6 (2020): 589-594.

9. D Bonn. "SARS virus in tears?" Lancet Infection Disease 4.8 (2004) 480 .

10. C Sun., et al. "Role of the Eye in transmitting human coronavirus: What we do not know". Frontiers in Public Health 8 (2020): 155.

11. L Jun., et al. "Assessing Viral Shedding and Infectivity of Tears in Coronavirus Disease". Ophthalmology 127.7 (2020): 977979.

12. European Centre for Disease Prevention and Control. Q\& A on COVID-19.

13. World Health Organization. Infection prevention and control during health care when novel coronavirus (nCoV) infection is suspected.

14. J Xia., et al. "Evaluation of coronavirus in tears and conjunctival secretions of patients with SARS-CoV-2 infection". Journal of Medical Virology 92 (2020): 589-594.

15. The Department of Health and Social Care (DHSC) and Public Health England (PHE), COVID-19: infection prevention and control guidance.

16. KY Lian., et al. "Infection control guidelines for optometrists 2016". Clinical and Experimental Optometry 100.4 (2017): 341-356

17. D Fonn and L Jones. "Hand hygiene is linked to microbial keratitis and corneal guidelines for optometrists 2016". Clinical and Experimental Optometry 100.4 (2017) 132-135. 
18. SSY Tseng. "SARS, avian flu, bioterror, infection control awareness for the optometrist". Clinical and Experimental Optometry 90.1 (2007) 31-35.

19. F Zeri and SA Naroo. "Contact lens practice in the time of COVID -19". Contact Lens and Anterior Eye (2020).

20. In- Office Disinfection of Multi-Patient use Diagnostic Contact Lenses. Angelica Polizzi. American Academy of Optometry Section on Cornea, Contact Lenses and Refractive Technologies (2020).

21. Contact lens Wear and Coronavirus guidance.

22. CDC. Coronavirus disease 2019 (COVID-19), Centre for Disease Control and Prevention (2020).

23. P Sankaridurg., et al. "Adverse events and infections: which ones and how many? In: D. Sweeney (Ed.), silicone hydrogels: Contiuous wear contact lenses". Butterworth-Heinemann, Oxford (2004): 217-274

24. D Sweeney., et al. "Clinical performance of silicone hydrogel lenses, in: D.sweeney (Ed.), silicone hydrogels: Contiuous wear contact lenses". Butterworth-Heinemann, Oxford (2004): 164216.

25. Taurakhia S., et al. "COVID-19 guidelines for optometry and optical services post-lockdown". Indian Journal of Ophthalmology 68 (2020): 1533-1539.

\section{Assets from publication with us}

- Prompt Acknowledgement after receiving the article

- Thorough Double blinded peer review

- Rapid Publication

- Issue of Publication Certificate

- High visibility of your Published work

Website: www.actascientific.com/

Submit Article: www.actascientific.com/submission.php

Email us: editor@actascientific.com

Contact us: +919182824667 